\title{
ZrCoCe Getter Film Solution for Under Controlled Atmosphere MEMS Packaging
}

\author{
Yaohua $\mathrm{Xu}^{1, \mathrm{a}}$, Jiandong Cui ${ }^{1, \mathrm{~b}}$, Hang Cui ${ }^{1, \mathrm{c}}$, Hao Zhou ${ }^{1, \mathrm{~d}}$, Zhimin Yang ${ }^{1, \mathrm{e}}$ and \\ Jun $\mathrm{Du}^{1, \mathrm{f}}$ \\ ${ }^{1}$ Advanced Electronic Materials Institute, General Research Institute for Nonferrous Metals, Beijing \\ 100088 , China \\ axyh1985620@126.com, 'buijiandong@grinm.com, ' hangcui@163.com, ${ }^{\mathrm{d}}$ zhouhao07@gmail.com, \\ epower@grinm.com, ${ }^{\mathrm{f}}$ dujun@grinm.com
}

Keywords: Non-evaporable getter; Thin films; ZrCoCe; DC magnetron sputtering; Sorption property; Vacuum packaging

Abstract. In order to specifically support the technology trend of increased miniaturization of Micro Electro Mechanical Systems (MEMS) devices, highly porous $\mathrm{ZrCoCe}$ non-evaporable getter (NEG) film has been produced by DC magnetron sputtering from a preformed $\mathrm{ZrCoCe}$ alloy target. The porous film is composed of columnar crystals, which is further built up with assembled $\mathrm{ZrCoCe}$ amorphous or nanocrystalline grains. Gas sorption investigation shows that this film can be activated at low temperature and exhibits excellent stable sorption characteristics. Sorption properties can be further improved with elevating the activation temperatures due to the microstructure modification. The capability of $\mathrm{ZrCoCe}$ films withstanding wafer physical or chemical cleaning processes is investigated, indicating their compatibility with MEMS vacuum packaging and the appropriate way to store them.

\section{Introduction}

Discrete packages are very critical to assure an excellent hermeticity in many MEMS devices to perform their basic functions properly and to enhance their reliability by keeping these devices away from the harmful external environment [1-4]. With the technology trend of increased miniaturization of MEMS devices, maintaining the needed vacuum level in such packages for the entire MEMS device life becomes a real technical challenge due to the very high surface to volume ratio [1]. The most common and technically accepted way to maintain a controlled ambient environment in a hermetically sealed MEMS device is to use a NEG capable of chemically absorbing active gasses, such as $\mathrm{H}_{2} \mathrm{O}, \mathrm{CO}, \mathrm{CO}_{2}, \mathrm{O}_{2}, \mathrm{~N}_{2}$ and $\mathrm{H}_{2}$. However, the conventional bulk or sheet getters having the property of high pumping speed even at room temperature, could not be used in these minimizing MEMS devices due to the restricted space [5-7]. It is urgently required to develop the highly porous thin-film-type getters with several $\mu \mathrm{m}$ thickness for the applications of MEMS vacuum devices.

$\mathrm{Ti}$, Ti-Zr-V, Zr-V-Fe or $\mathrm{ZrV}_{2}$ are typical NEG film materials, which have been widely studied in sealed-off vacuum technology. Nevertheless, the aforementioned materials may be defined as NEG materials of specific use, and are often described and referred to in patents and technical or commercial bulletins with reference to their use in specific applications [8-10]. Zr-Co-rare earth (RE) alloys are specially developed to fulfil some specific integration needs. These alloys are advantageous because they are suitable for general use. they have a relatively low activation temperature $200-500^{\circ} \mathrm{C}$, are capable of sorbing a wide variety of gases, and minimize the environmental and safety risks compared with known nonevaporable getter alloys [11].

It is known from previous studies that $\mathrm{ZrCoRE}$ films have been prepared by RF magnetron sputtering and sorption properties can be improved by optimizing related parameters [12,13]. However, the performance of the above films are too low to fulfil practical applications and mass production. More efficient sorption capacity and higher stability are imminently required. In this paper, the highly porous $\mathrm{ZrCoCe} \mathrm{NEG}$ film has been successfully produced by DC magnetron sputtering from a preformed $\mathrm{ZrCoCe}$ alloy target. The detailed results of the characterization of 
surface morphology and microstructure of the porous film will be first presented. The gas sorption performance of the porous film is also discussed in detail.

\section{Experimental}

The $\mathrm{ZrCoCe}$ getter film was fabricated on (100) Si substrate by DC magnetron sputtering process. The substrate was prepared in accordance with the standard RCA cleaning method. A ZrCoCe alloy target $\left(\mathrm{Zr}_{76.2} \mathrm{Co}_{20.7} \mathrm{Ce}_{3.1}\right.$ at.\%, purity $\left.>99.5 \%, \Phi 60 \times 5 \mathrm{~mm}\right)$ was made by vacuum melting and machining. To decrease gas contamination, the base pressure of deposition chamber was less than 1.0 $\times 10^{-6} \mathrm{~Pa}$. High purity Ar gas (purity $\geq 99.999 \%$ ) was used as sputtering gas and the working Ar pressure is about $3 \mathrm{~Pa} .100 \mathrm{~W}$ DC power was used to facilitate the sputtering process with the film deposition rate of $55-65 \mathrm{~nm} / \mathrm{min}$. The substrate was baked by a built-in heater to provide a steady deposition temperature of $200{ }^{\circ} \mathrm{C}$ during depositing. The distance between target and substrate and glancing angle were $6 \mathrm{~cm}$ and $70^{\circ}$, respectively.

$\mathrm{H}_{2}$ sorption measurement of samples was carried out by dynamic sorption method according to ASTM F798-97 [14]. A Hitachi S-4800 field emission scanning electron microscope (Tokyo, Japan) with an integrated energy dispersive X-ray spectrometer (EDX) was used to analyze the composition, morphology and cross-sectional structure of the film. The EDX operating voltage is $20 \mathrm{kV}$. The microstructure and phase of the film were determined by X-ray diffraction (D/MAX2200, Rigaku, Japan) operated at $36 \mathrm{kV}$ and $20 \mathrm{~mA}$. The Brunauer-Emmett-Teller surface area of the $\mathrm{ZrCoCe}$ film was measured by recording nitrogen adsorption/desorption isotherms using a Micromeritics ASAP 2010 instrument (Micromeritics Instrument Corporation, USA).

\section{Results and discussion}

Microstructure Properties of ZrCoCe Film. The gas-absorption performance of the onevaporable film getter is strongly related to the morphologies and microstructures of the films. High roughness and porosity induce an increase of both surface gas capacity and sticking probability. The former is simply due to the larger surface area, while the latter is caused by multiple collisions of the impinging molecules inside the surface pores $[4,7,12]$.

Fig. 1 displays the the SEM micrographs of the surface and cross-section and EDS pattern of the porous $\mathrm{ZrCoCe}$ film on Si substrate, respectively. It shows that the porous $\mathrm{ZrCoCe}$ film is composed of isolated columns, forming finely packed nanocrystals at the surface, as shown in Fig. 1 (a)-(c). These finely packed nanocrystals give rise to high density grain boundaries and the rough surface of the film, and hence leads to the high specific surface area of $63 \mathrm{~m} 2 / \mathrm{g}$. The typical width of columns and inter-distance between the columns are $250 \mathrm{~nm}$ and $40 \mathrm{~nm}$, respectively. Fig. 1 (d) shows the energy dispersive X-ray diffraction pattern of the porous TiZrV film. The average composition of the porous $\mathrm{ZrCoCe}$ film is $\mathrm{Zr} 77.8$ at.\%, Co 20.7 at.\%, and Ce 1.5 at.\% according to the EDS analysis.

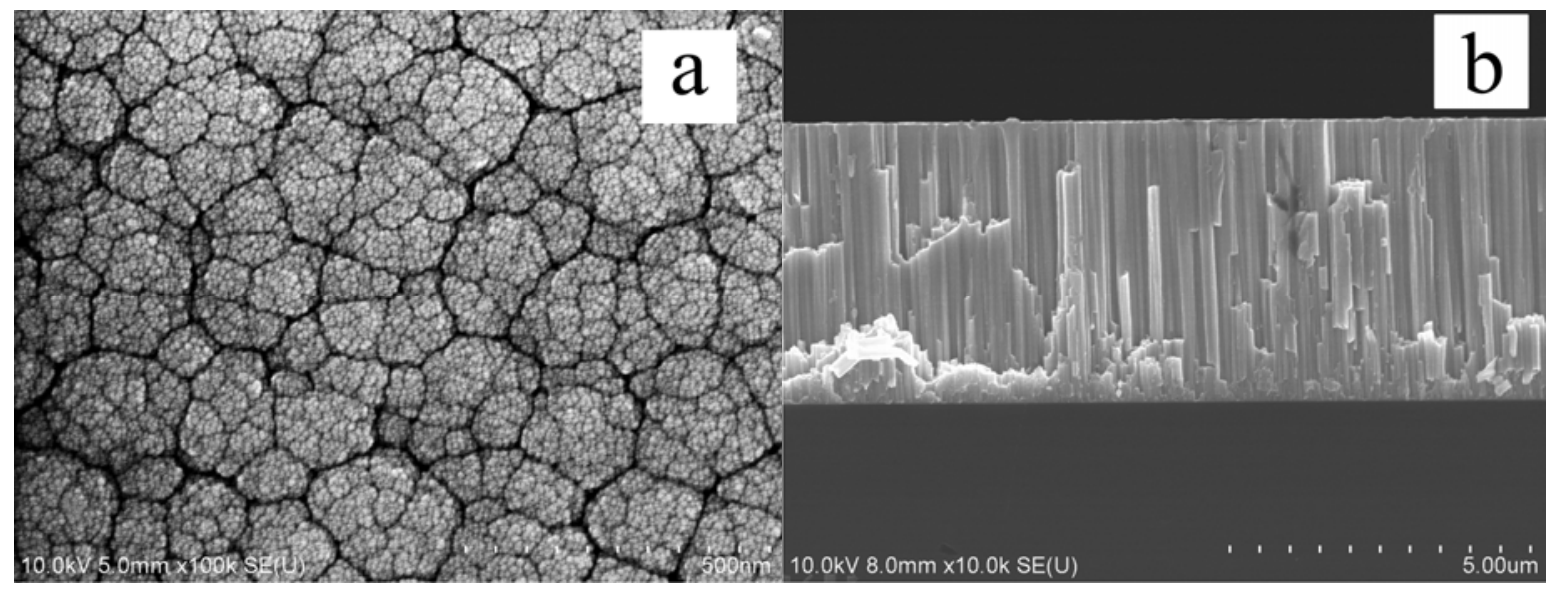




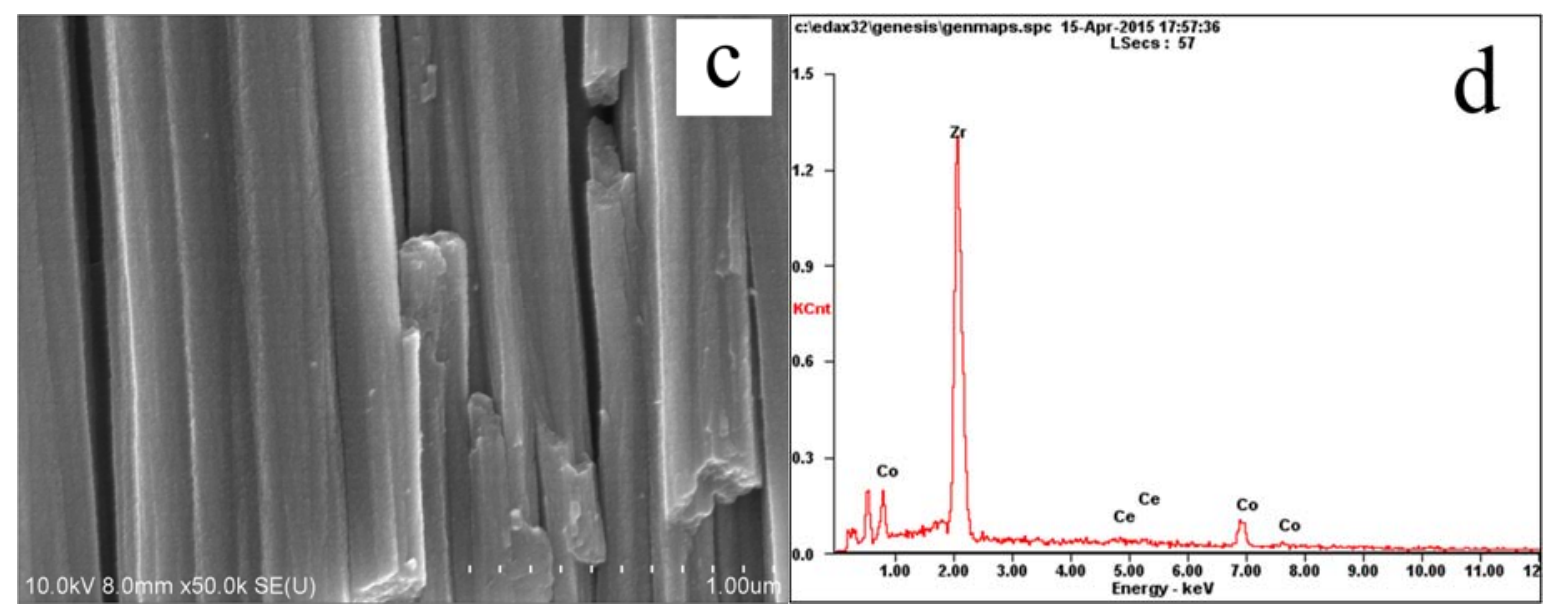

Fig. 1. SEM images and EDS pattern of the porous $\mathrm{ZrCoCe}$ film.

Effects of Activation Procedure on Sorption Property. It is known that the activation and gas sorption properties of an NEG depend strongly on active surface areas, diffusion process and matrix solubility $[15,16]$. During activation process, the NEG surface is progressively reduced by heating to a temperature high enough to facilitate the diffusion of the passivation layer covering the surface of each getter particle into the getter bulk, resulting in highly active surface.

Fig. 2 shows the hydrogen sorption properties of $\mathrm{ZrCoCe}$ film at room temperature after activated at different temperatures for $30 \mathrm{~min}$. It can be seen that activation temperature can greatly affect the sorption properties of the porous $\mathrm{ZrCoCe}$ film. Both sorption speed and capacity of the film increases with elevating the activation temperature from $250{ }^{\circ} \mathrm{C}$ to $400{ }^{\circ} \mathrm{C}$. For activation temperature above $300{ }^{\circ} \mathrm{C}$, the $\mathrm{ZrCoCe}$ film exhibits excellent sorption properties with a broad plateau on the plot of sorption speed versus quantity (curve $\mathrm{b}-\mathrm{d}$ ). When the activation temperature is $250{ }^{\circ} \mathrm{C}$, the initial sorption speed of the film is low due to the surface not fully activated (curve a) [12]. When the activation temperature raises to $300^{\circ} \mathrm{C}$, more active and cleaner surface is obtained, resulting in a better initial sorption speed of $87 \mathrm{~cm}^{3} \cdot \mathrm{s}^{-1} \cdot \mathrm{cm}^{-2}$ (curve b). This value will be further elevated and reachs 180 and $236 \mathrm{~cm}^{3} \cdot \mathrm{s}^{-1} \cdot \mathrm{cm}^{-2}$, corresponding to the activation temperature of $350{ }^{\circ} \mathrm{C}$ and $400{ }^{\circ} \mathrm{C}$, respectively.

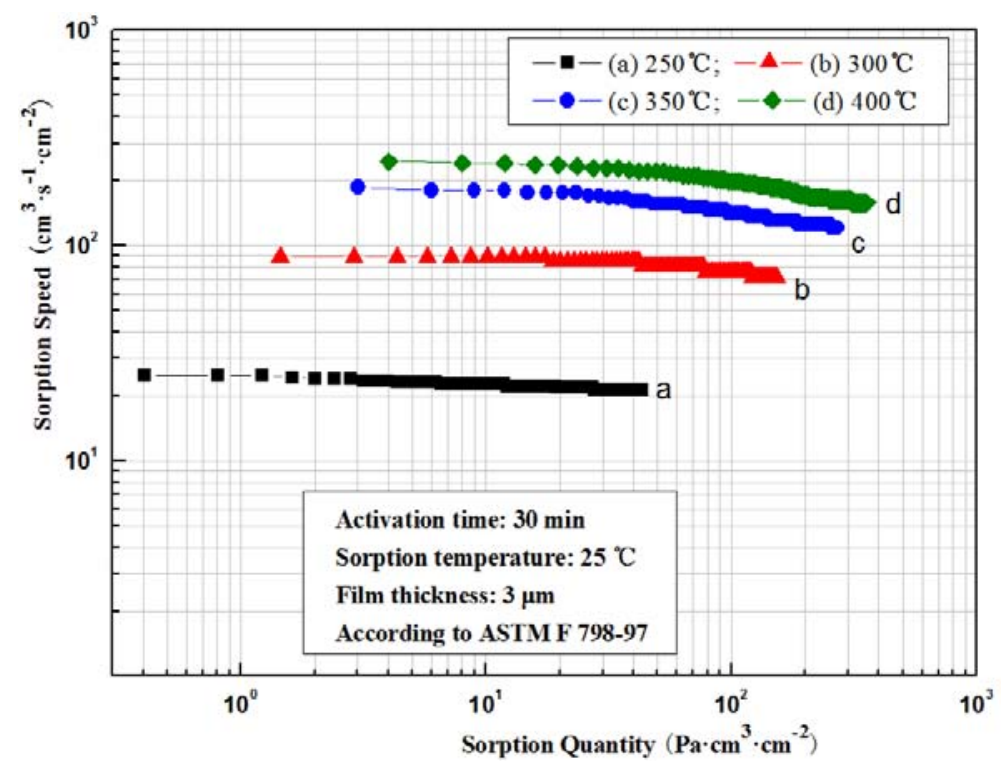

Fig. 2 Sorption characteristics of the $\mathrm{ZrCoCe}$ film after different activation temperatures for $30 \mathrm{~min}$.

Effects of Activation Procedure on Microstructure Property. As is known to all, activation mechanism of getter materials is related to desorption of adsorbed gas molecules, decomposition of oxides and diffusion of carbon and oxygen from the surface into the bulk or surface reduction of metallic atoms [12-14]. Meanwhile, microstructures of the getter will also change during activation 
process, especially thin film getters. Fig. 3 displays the XRD patterns of the ZrCoCe films after a variety of activation treatments. As can be seen, one broad diffraction peak with low intensity at around $36^{\circ}$ appears for both as-deposited and low-temperature activated $\left(250\right.$ or $\left.300{ }^{\circ} \mathrm{C}\right)$ films, implying that the $\mathrm{ZrCoCe}$ film is composed of amorphous or nanocrystalline grains, which are dispersed in the amorphous matrix. By analyzing the full width at half-maximum (FWHM) of the (110) diffraction peak according to Scherrer's formula calculation, the average crystallite size of the film is about $2 \mathrm{~nm}$. These fine crystallites increase the diffusing channel of gas molecules, which accelerates the surface gas diffusion and improves the gas sorption capacity of the film [16]. When the activation temperature increases, nanocrystallines grow up and amorphous regions crystallize indicated by a decrease of the FWHM and an increase of the intensity of XRD peaks, shown in Fig. 3. After activated at $400{ }^{\circ} \mathrm{C}$ for $30 \mathrm{~min}$, the $\mathrm{ZrCoCe}$ film mainly exhibits body-centered-cubic structure of $\alpha$-Zr phase with (110), (101) and (200) crystal planes. Moreover, after activation, the orthorhombic $\mathrm{Zr}_{3} \mathrm{Co}$ phase with diffraction peaks of (131) and (113), which is favorable for absorbing the hydrogen gas also appeared.

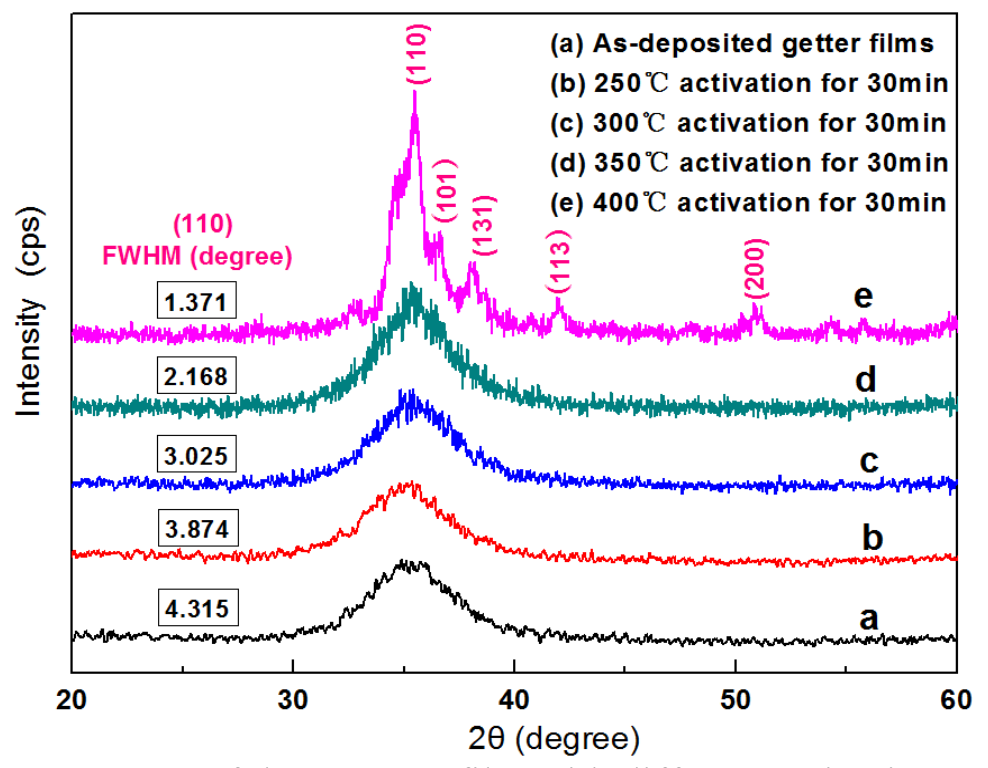

Fig. 3. XRD patterns of the $\mathrm{ZrCoCe}$ film with different activation procedures.

Effects of Wafer Cleaning Processes on Sorption Property. It is known that the capability of getter films withstanding wafer cleaning processes is of great importance because it is related to whether good compatibility with the integration process of MEMS devices [1]. In order to evaluate the reliability of the porous $\mathrm{ZrCoCe}$ film, hydrogen sorption properties of the $\mathrm{ZrCoCe}$ film after different wafer cleaning processes were investigated. To begin with, the film was previously treated with isopropanol, acetone, chlorobenzene, argon ion sputtering for $1 \mathrm{~min}$, respectively. The detailed results are shown in Fig. 4. It clearly shows that sorption properties of the treated $\mathrm{ZrCoCe}$ film increase after the cleaning process of isopropanol and argon ion sputtering, shown as curves b and c, respectively. However, an opposite effect will be obtained for acetone or chlorobenzene, demonstrating an obvious decline on the plot of sorption speed versus quantity (curves $d$ and e). Compared with acetone, standard wafer chemical cleaning with chlorobenzene could led to a more serious sorption decrease and even the loss of gettering ability, implying chlorobenzene not an ideal cleaning solvent for this $\mathrm{ZrCoCe}$ film. Additional studies are necessary to substantiate this argument.

According to the above results, it can be said that such $\mathrm{ZrCoCe}$ film has good capability of cleaning resistance, and can be well compatible with MEMS vacuum packaging. In addition, it provides an important reference for the storage of NEG films, and further studies are in progress and will be presented later. 


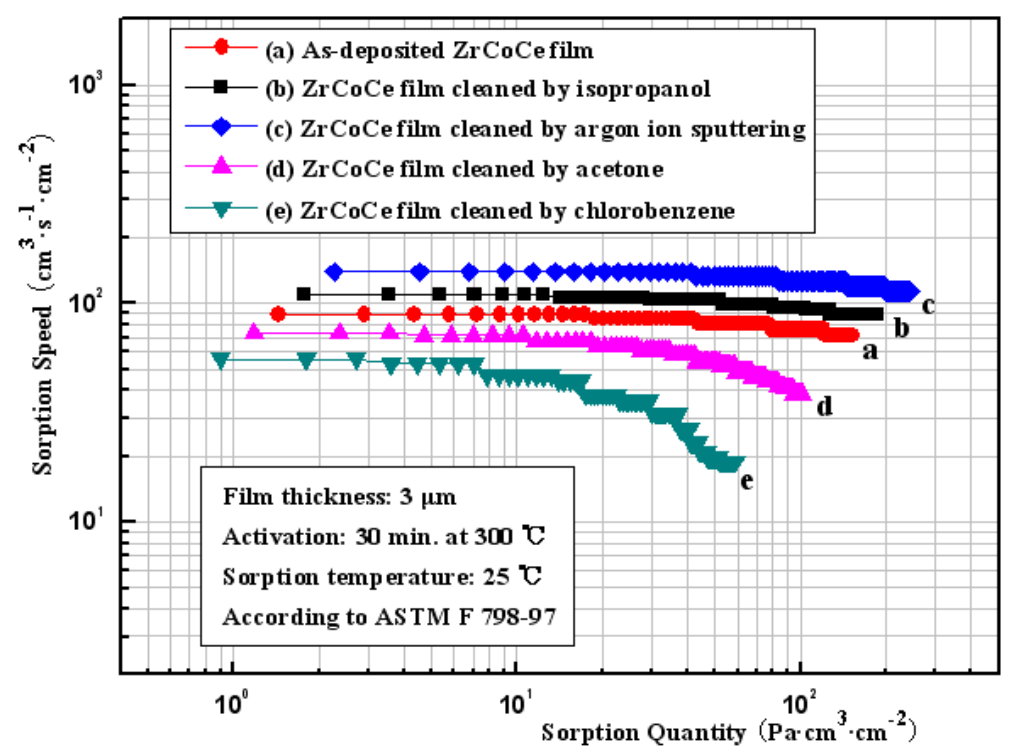

Fig. 4. Sorption characteristics of the $\mathrm{ZrCoCe}$ film after different wafer cleaning processes.

\section{Conclusions}

1) Highly porous $\mathrm{ZrCoCe} \mathrm{NEG}$ film with columnar structure on (100) Si wafers has been successfully fabricated by DC magnetron sputtering. The porous film is mainly composed of amorphous or nanocrystalline $\mathrm{ZrCoCe}$ grains with a high specific surface area of $63 \mathrm{~m}^{2} / \mathrm{g}$.

2) The porous $\mathrm{ZrCoCe}$ film can be activated at low temperatures in range of $250-300^{\circ} \mathrm{C}$ and demonstrates excellent stable hydrogen sorption properties. Sorption properties can be further improved with elevating the activation temperatures due to the microstructure modification.

3) The capability of the porous $\mathrm{ZrCoCe}$ film withstanding wafer cleaning processes was evaluated, indicating its potential applications in MEMS vacuum packaging.

\section{References}

[1] Y.F. Jin, Z.F. Wang, L. Zhao, P.C. Lim, J. Wei and C.K. Wong: J Micromech Microeng Vol. 14 (2004), p. 687

[2] R. Ramesham and R.C. Kullberg: J Micro/Nanolith MEMS MOEMS Vol. 8 (2009), p.031307

[3] J. Hidalgo, P. Poulakis, J. Kohler, J. Del-Cerro and A. Barrientos: Sensors Vol. 12 (2012), p. 2219

[4] L. Tenchine, X. Baillin, C. Faure, P. Nicolas and E. Martinez: Procedia Engineering Vol. 5 (2010), p. 359

[5] C. Benvenuti, P. Chiggiato, F. Cicoira, Y. L’Aminot and V. Ruzinov: Vacuum Vol. 73 (2004), p. 139

[6] C. Bemard, E. Blanquest and M. Pons: Surf Coat Tech Vol. 202 (2007), p. 790

[7] C.C. Li, J.L. Huang, R.J. Lin, D.F. Li and C.H. Chen: J. Vac. Sci. Technol Vol. 25 (2007), p. 1373

[8] C. Vivek, L. Xie and B.T. Chen: J Electron Mater Vol. 42 (2013), p. 485

[9] C.C. Li, J.L. Huang, R.J. Lin, H.K. Chang and J.M. Ting: Surf Coat Tech Vol. 200 (2005), p. 1351

[10]C. Benvenuti, P. Chiggiato, P. Costa Pinto, A. Escudeiro Santana, T. Hedley, A. Mongelluzzo, V. Ruzinov and I. Wevers: Vacuum Vol. 60 (2001), p. 57

[11]C. Boffio, A. Corazza and S. Tominetti, U.S. Patent 5,961,750. (1999)

[12]J.G. Bu, C.H. Mao, Y. Zhang, X.Y. Wei and J. Du: J Alloy Compd Vol. 529 (2012), p. 69 
[13]D. Petti, M. Cantoni, M. Leonea, R. Bertaccoa and E. Rizzi: Appl Surf Sci Vol. 256 (2010), p. 6291

[14]798-97 ASTM, Standard Practice for Determining Gettering Rate, Sorption Capacity, and Gas Content of Non-Evaporable Getters in the Molecular Flow Region, 2002.

[15]C. Benvenuti, P. Chiggiato, P. Costa Pinto, A. Prodromides and V. Ruzinov: Vacuum Vol. 71 (2003), p. 307

[16]A. Moghadam Heidary, V. Dashtizad, A. Kaflou and H. Yoozbashizadeh: Vacuum Vol. 111 (2015), p. 9 